\title{
Angiographic demonstration of incoordinate motion of the ventricular wall after the Fontan operation
}

\author{
Daniel J Penny, Andrew N Redington
}

\begin{abstract}
Objective-To study regional wall motion of the systemic ventricle in patients after the Fontan operation.

Design and patients-Systemic ventricular angiograms of 15 patients after the Fontan operation and of 11 unoperated patients with a univentricular atrioventricular connection were digitised frame by frame. Strict criteria for abnormal wall motion were used so that minor abnormalities were not considered.

Results-Incoordinate contraction of the ventricular wall was found in five of the 11 patients before and in four of the 15 patients after the Fontan operation (NS). Only three of the 11 patients before the Fontan operation, showed incoordination of ventricular relaxation, but incoordinate relaxation was seen in 12 of the 15 patients after operation $(p<0.01)$.

Conclusions-Whereas incoordination of ventricular contraction was common in patients with a univentricular atrioventricular connection, before or after the Fontan operation, incoordinate relaxation of the ventricular wall was a common consequence of the Fontan operation and was rare in patients before operation.
\end{abstract}

The assessment of systemic ventricular function in patients after the Fontan operation ${ }^{1}$ has principally relied on the assessment of global systolic pump activity. ${ }^{23}$ The indices used have been based on the extrapolation of the contractile properties of single myocardial fibres to the intact heart, ${ }^{2}$ and it has been assumed that any derangement in such indices might reflect abnormalities of ventricular "contractility". The use of these indices, however, neglects potentially important regional abnormalities of timing and function, which may have important implications for ventricular pressure development, ${ }^{4}$ relaxation, ${ }^{56}$ and diastolic filling, ${ }^{7}$ even when the functional properties of the individual myofibrils are normal. It follows therefore that any abnormalities in measured indices of global ventricular contraction or filling cannot be ascribed to abnormal ventricular "contractility" or "compliance" unless abnormalities of regional function have been excluded.

It is thus timely to extend the assessment of systemic ventricular function in patients after the Fontan operation beyond the analysis of global pump function and to include the study of regional variation in ventricular wall movement. This is particularly pertinent to these geometrically unusual ventricles that are subjected to profound changes in loading conditions at the time of operation.

We have previously described the presence of abnormal intraventricular Doppler flow in patients after the Fontan operation, ${ }^{8}$ and have proposed that the presence of this intraventricular flow, as in other patients with left ventricular disease, ${ }^{910}$ may reflect the presence of regional abnormalities of ventricular function. A direct assessment of regional ventricular function, however, has not been performed in this patient group. In this study we have attempted such an assessment by frame by frame analysis of ventricular angiograms before and after the Fontan operation in patients with a univentricular atrioventricular connection.

\section{Methods}

PATIENTS

We studied 26 patients. Fifteen patients with a median age of 104 (range 43-196) months were studied at a median interval of $17(5-54)$ months after the Fontan operation. Of these, nine had tricuspid atresia with a concordant ventriculoarterial connection. Five patients had double inlet left ventricle (with a discordant ventriculoarterial connection in three, a concordant ventriculoarterial connection in one, and pulmonary atresia in one). One patient had pulmonary atresia with an intact ventricular septum. Atriopulmonary anastomosis had been performed in 12, and in two the rudimentary right ventricle was included in the anastomosis, with closure of the ventricular septal defect. One patient with double inlet left ventricle and a restrictive ventricular septal defect underwent the Damus-KayeStansel procedure at the time of the Fontan operation. At the time of study all patients were in functional class I or II and none had evidence of significant atrioventricular valve regurgitation on angiography. One patient had a restrictive ventricular septal defect, which was subsequently enlarged.

Eleven patients with a univentricular atrioventricular connection were studied before the Fontan operation at a median age of 51 (35-160) months. Of these, six had tricuspid atresia (with a concordant ventriculoarterial connection in five and a discordant

\author{
ational Heart and Lung \\ Hospital, Sydney Street, \\ Accepted for publication \\ 1 August 1991 \\ Department of \\ A N Redington \\ Dr Andrew N Redington, \\ Department of Paediatric
}


ventriculoarterial connection in one). Four patients had double inlet left ventricle (with a discordant ventriculoarterial connection in two and double outlet left ventricle in two). One patient had double inlet right ventricle with a double outlet ventriculoarterial connection. Five patients had undergone insertion of one or more systemic to pulmonary artery shunts, three had undergone banding of the pulmonary artery alone, and one patient had undergone banding of the pulmonary artery, before insertion of a systemic to pulmonary artery shunt.

\section{VENTRICULOGRAMS}

Cardiac catheterisation was performed during general anaesthesia. Orthogonal biplane ventricular angiograms were recorded on $35 \mathrm{~mm}$ cinefilm at a frame rate of 50 frames a second. Angiographic projections were chosen to display the interventricular septum (direct anteroposterior and lateral when the rudimentary ventricle was to the left of the dominant ventricle and $30^{\circ}$ right anterior oblique $60^{\circ}$ left anterior oblique when the rudimentary ventricle was to the right). A mark coinciding with the $R$ wave of the electrocardiogram was automatically recorded on the cinefilm.

\section{ANALYSIS}

Extrasystoles and post-extrasystoles were not included in the analysis. Cavity outlines were manually digitised frame by frame on a Summagraphics digitising plate interfaced with a Prime 750 mainframe by methods previously described. ${ }^{11}$ A fixed external reference point was used throughout.

We assessed regional wall motion by constructing isometric and contour plots for each beat as described by Gibson and coworkers. ${ }^{11}$ The technique in essence provides multiple plots of wall motion against time derived from 40 equally spaced segments around the boun-

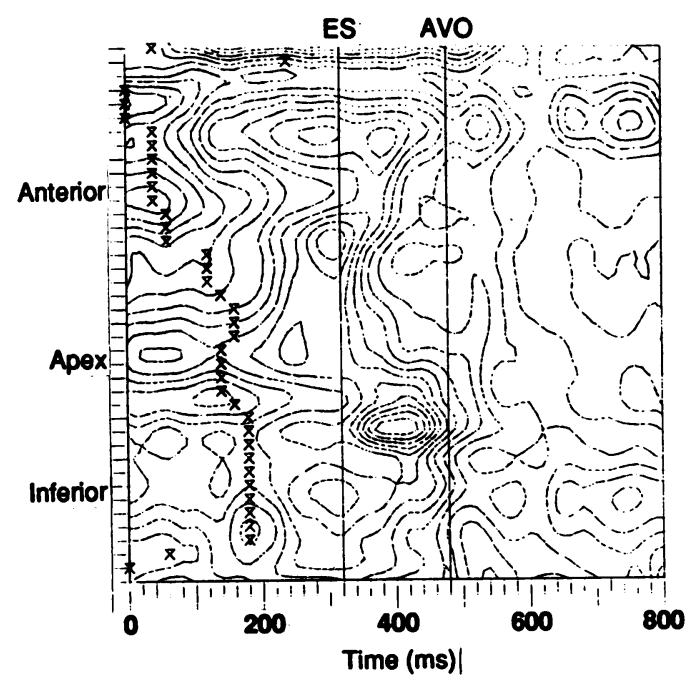

Figure 1 Contour display of regional wall movement of the left ventricle in a patient after Fontan operation. Onset of inward movement of much of the inferior surface of the ventricular wall is delayed until $180 \mathrm{~ms}$ after the onset of electromechanical systole. Time 0 on the horizontal axis marks the $R$ wave on the electrocardiogram. X, onset of inward movement of each segment of the ventricular wall; $E S$, time of end systole (which is taken as minimum cavity dimension); $A V O$, time of atrioventricular wall opening.

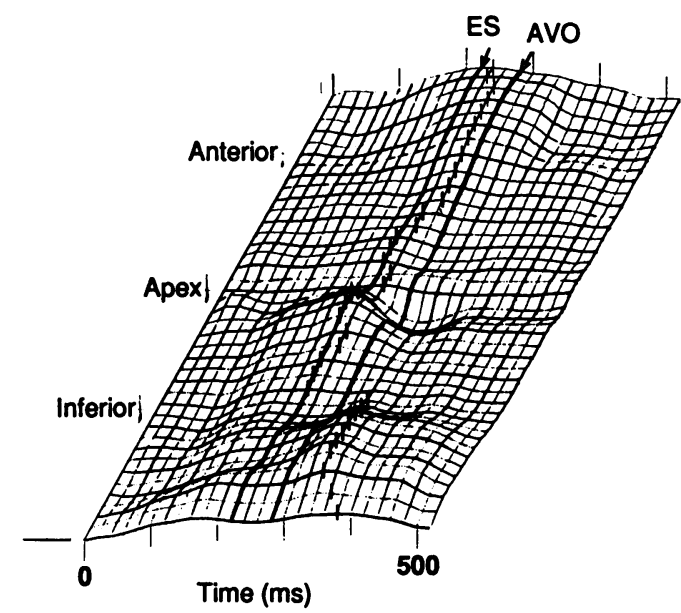

Figure 2 Isometric display of regional wall movement in a patient after the Fontan operation. Peak inward movement of a segment of the ventricular wall is denoted by a short vertical line. There is continued inward movement of much of the inferior border of the ventricle until $60 \mathrm{~ms}$ after the atrioventricular valve opens.

Isovolumic relaxation time is taken as the time between end systole and atrioventricular valve opening. See legend to fig 1 for abbreviations.

dary of the end diastolic frame such that segments 1-15 represent the inferior wall of the ventricle, segments $15-25$ the apical region, and segments 25-40 the free wall of the ventricle. Regional wall motion can then be represented by either stacking these individual plots obliquely to give an isometric display (fig 1), or by the construction of a series of contours that represent inward or outward movement of the endocardium in $1 \mathrm{~mm}$ steps from its end diastolic position (fig 2). It is also possible to construct two-frame displays of the ventricular outline at designated times during the cardiac cycles (fig 3 ).

CRITERIA

Strict criteria for abnormal wall motion were

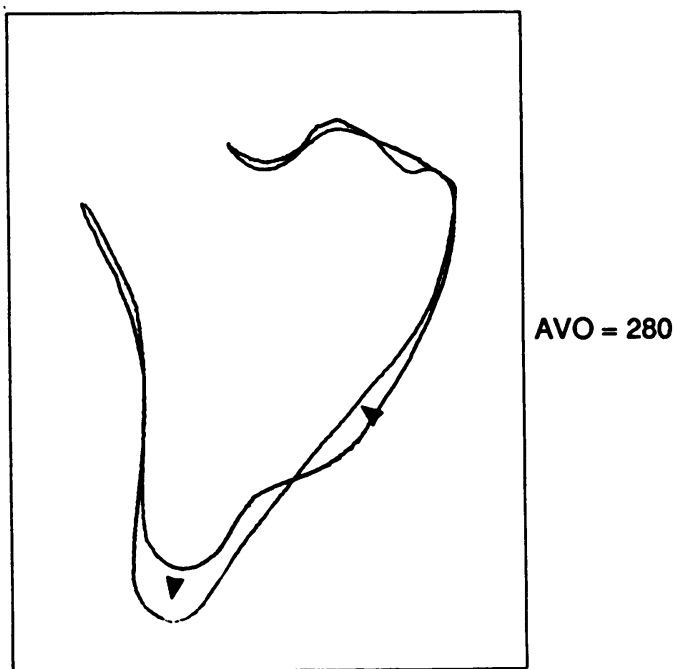

Frames at 200 and $280 \mathrm{~ms}$

Figure 3 Two frame display of ventricular wall movement during isovolumic relaxation in a patient (not one of those providing data for figs 1 and 2) after the Fontan operation. Frames are taken at end systole (minimum cavity dimension $200 \mathrm{~ms}$ ) and at atrioventricular valve opening (AVO) $(280 \mathrm{~ms})$. There atrioventricular valve opening ( $A V O)(280 \mathrm{~ms})$. There outward movement of the apex. 
used, so that minor abnormalities were not considered.

Incoordinate contraction was defined as: (a) outward movement of four adjacent segments between the onset of inward motion and minimal cavity size (dyskinesia). (b) Onset of inward movement of four adjacent segments occurring more than $140 \mathrm{~ms}$ after the QRS complex (timing abnormality).

Relaxation abnormalities were defined as: (a) inward movement of more than $50 \%$ of total inward movement of four adjacent segments after minimal cavity dimension. (b) Outward movement of more than $50 \%$ of total outward movement of four adjacent segments before mitral valve opening.

\section{Results}

SYSTOLIC ABNORMALITIES

Regional abnormalities of ventricular contraction were detected in five of the 11 patients with a univentricular atrioventricular connection before the Fontan operation. In one patient with tricuspid atresia this consisted of a discrete area of apical dyskinesis and in four (three of whom had tricuspid atresia and one a double inlet ventricle) a regional delay in the onset of inward wall movement was detected. Incoordinate ventricular contraction was found in four of the 15 patients after the Fontan operation. This difference was not statistically significant. A discrete area of inferoapical hypokinesia was found in two, and localised timing abnormalities in two (fig 1 ).

\section{RELAXATION ABNORMALITIES}

Whereas regional abnormalities of ventricular relaxation were detected in only three of the 11 patients before the Fontan operation (two with tricuspid atresia and one with double inlet right ventricle), incoordinate relaxation was found in 12 of the 15 patients after operation $(p<0.01)$. This usually consisted of large areas of inward movement of the ventricular wall in the inferoapical segments after minimal cavity dimension associated with excessive outward movement of another region (figs 2 and 3 ).

One death occurred after the Fontan operation in the patients for whom preoperative data were available. This patient had double inlet left ventricle and showed no regional abnormalities of function at angiography. In the remaining patients before operation the presence of regional abnormalities of function was not a predictor of the duration of stay on the intensive care unit after subsequent Fontan operation.

\section{Discussion}

Previous workers have documented an apparent improvement in "ventricular function" after the Fontan operation. " These studies, however, have been restricted to the use of global indices of ventricular systolic pump function only. It is now well recognised that any attempt to provide a comprehensive assessment of ventricular function must extend beyond the use of such indices, and include both the assessment of ventricular diastolic function and the study of regional abnormalities of ventricular wall motion. This is the first study to attempt such an analysis in patients after the Fontan operation.

Two distinct types of abnormality were seen. Some patients had major regional abnormalities of ventricular contraction, whereas nonuniformity of ventricular relaxation was found in others.

Incoordinate ventricular contraction was seen in five $(45 \%)$ of the 11 patients with a univentricular atrioventricular connection before the Fontan operation, and in four $(27 \%)$ of the 15 patients after operation. The presence of regional abnormalities of ventricular systolic function has been described in patients with a univentricular atrioventricular connection by Gibson et $a l^{12}$ They found regional delays in the onset of contraction and areas of hypokinesia in 11 of 20 patients with a univentricular atrioventricular connection, although the study did not include patients after the Fontan operation. Incoordinate ventricular contraction may adversely affect ventricular pressure development during early systole and thus influence socalled indices of ventricular contractility, such as Vmax, even when the contractile properties of the individual myofibrils are normal.

The factors responsible for these abnormalities are unknown but may be related to abnormalities of myofibril architecture and orientation, ${ }^{13}$ chronic volume overload of the dominant ventricle, or hypoxaemia that result from the presence of a univentricular atrioventricular connection. Certainly, in other groups of patients, chronic volume overload has been shown to be a powerful precipitant of regional abnormalities of ventricular function. ${ }^{14}$ The relative frequency of these systolic abnormalities seems not to be influenced by the Fontan operation. Global systolic ventricular function is also unchanged or improved ${ }^{2}$ after the Fontan operation and these data emphasise that, in the short term at least, systolic main chamber performance is unlikely to be a primary determinant of outcome after this operation.

The occurrence of regional abnormalities of ventricular relaxation, however, differed considerably between the two patient groups. Twelve $(80 \%)$ of the 15 patients after the Fontan operation but only three $(27 \%)$ of 11 patients with a univentricular atrioventricular connection before the Fontan operation displayed such abnormalities $(p<0.01)$. These findings are consistent with our previous Doppler study, which documented the presence of intraventricular Doppler flow during isovolumic relaxation in $80 \%$ of patients after the Fontan operation but in none of 12 preoperative patients. ${ }^{8}$ Thus during isovolumic relaxation, while the atrioventricular and semilunar valves are closed and the fixed volume ventricle is attempting to relax, large areas of the ventricular wall continue to move inwards, associated with simultaneous outward movement of other regions.

These regional abnormalities of ventricular 
relaxation may have profound implications for ventricular relaxation and filling. Previous studies have described a prolongation of the time constant of relaxation, 5 and of isovolumic relaxation time ${ }^{6}$ and a reduction in early diastolic ventricular filling ${ }^{7}$ in patients with incoordinate ventricular relaxation. Of possible relevance to this are our previous observations of a prolonged isovolumic relaxation time and a reduction in the velocity of early rapid ventricular filling in patients after the Fontan operation compared with control or preoperative patients. $^{8}$

The precise mechanisms responsible for the widespread development of incoordinate relaxation of the systemic ventricle in patients after the Fontan operation are unclear. Previous studies, however, have shown the development of incoordinate ventricular relaxation in other groups of patients, after acute reductions in ventricular preload. ${ }^{15}$ Further studies have found evidence of incoordinate ventricular relaxation in patients with ventricular hypertrophy. ${ }^{10}$ The Fontan operation imposes a rapid and profound reduction in ventricular preload on a chronically volume overloaded ventricle. Our own findings suggest that large reductions in ventricular cavity dimension associated with pronounced increases in posterior wall thickness occur acutely after this operation. ${ }^{16}$ It is interesting to speculate that the regional abnormalities of ventricular relaxation that we found may be related at least in part to these findings.

In summary, regional abnormalities of ventricular contraction are common before and after the Fontan operation in patients with a univentricular atrioventricular connection. They may have important, so far unexplored, implications for interventricular pressure development and ventricular systolic function in these patients. Regional abnormalities of ventricular relaxation commonly develop in patients after the Fontan operation. As yet the mechanisms responsible for these are unclear. They may affect ventricular diastolic function, however, even in the presence of normal relaxation and stress strain properties of the individual myofibrils.
Abnormalities in the indices of global ventricular function must be interpreted with caution in these patients.

DJP is suported by the British Heart Foundation.

1 Fontan F, Baudet E. Surgical repair of tricuspid atresia. Thorax 1971;26:240-8.

2 Gewillig MH, Lundstrom UR, Deanfield JE, Bull C, Franklin RC, Graham TP, et al. Impact of Fontan operation on left ventricular size and contractility in tricuspid atresia. Circulation 1990;81:118-27.

3 Hagler DJ, Seward JB, Tajik J, Ritter DG. Functional assessment of the Fontan operation: combined M-mode, two-dimensional and Doppler echocardiographic studies. J Am Coll Cardiol 1984;4:756-64.

4 Gibson DG. Ventricular function. In: Anderson RH, Macartney FJ, Shinebourne EA, Tynan M, eds. Paediatric cardiology. London: Churchill Livingstone, Paediatric cardic

5 Aoyagi $T$, lizuka $M$, Takahashi $T$, Ohya $T$, Serizawa $T$, Momomura $S$, et al. Wall motion asynchrony prolongs time constant of left ventricular relaxation. Am J Physio 1989;257:H883-90.

6 Ludbrook PA, Byrne JD, Tieffenbrunn AJ. Association of asynchronous protodiastolic segmental wall motion with impaired left ventricular relaxation. Circulation 1981; 64:1201-11.

7 Perrone-Filardi P, Betocchi S, Giustini G, Piscoine F, Indolf $\mathrm{C}$, Salvatore $M$, et al. Influence of left ventricular asynchrony on filling in coronary artery disease. $A m$ Cardiol 1988;62:523-7.

8 Penny DJ, Rigby ML, Redington AN. Abnormal patterns of intraventricular flow and diastolic filling after the Fontan operation: evidence for incoordinate ventricular wall motion. Br Heart J 1991;66:375-8.

9 Garrahy PJ, Kwan OL, Booth DC, De Maria AN. Assessment of abnormal systolic intraventricular flow patterns by ment of abnormal systolic intraventricular flow patterns by
Doppler imaging in patients with left ventricular dyssDoppler imaging in patients with

10 Sasson Z, Hatle L, Appleton CP, Jewett M, Alderman EL, Popp RA. Intraventricular flow during isovolumic relaxation: description and characterisation by Doppler tion: description and characterisation by Doppler

11 Gibson DG, Prewitt RA, Brown DJ. Analysis of left ventricular wall movement during isovolumic relaxation ventricular wall movement during isovolumic relaxation
and its relation to coronary artery disease. Br Heart $J$ and its relation to.

12 Gibson DG, Traill TA, Brown DJ. Abnormal ventricular function in patients with univentricular heart. Herz 1981;4:226-31.

13 Becker AE, Caruso G. Congenital heart disease-a morphologists view on myocardial dysfunction. In: Becker AE, Losekoot TG, Marcelletti C, Anderson RH, eds. Paediatric cardiology vol 3. London: Churchill Livingstone, 1981:307-23.

4 Di Donato M, Barletta A, Mori F, Fantini F. Regional left wall motion abnormalities in chronic volume overload. Cathet Cardiovasc Diagn 1983;9:453-62.

15 Hall RJC, Doran J, McHaffie D, Gibson DG. The effect of nitroglycerin, beta-blockade with acebutolol and isometric stress on incoordinate left ventricular function. Eur Heart $J$ 1982;3:23-8.

16 Penny DJ, Lincoln C, Xiao H, Rigby ML, Redington AN. Acute effects of transition to a Fontan circulation [abstract]. Br Heart J 1991;66:66. 